\title{
Synthesis and Control of the Shell Thickness of Polyaniline and Polypyrrole Half Hollow Spheres Using the Polystyrene Cores
}

\author{
Su-Ryeon Yun, ${ }^{1}$ Gyeong-Ok Kim, ${ }^{1}$ Chan Woo Lee, ${ }^{2}$ Nam-Ju Jo, ${ }^{3}$ \\ Yongku Kang, ${ }^{4}$ and Kwang-Sun Ryu ${ }^{1}$ \\ ${ }^{1}$ Department of Chemistry, University of Ulsan, Daehak-ro 93, Nam-gu, Ulsan 680-749, Republic of Korea \\ ${ }^{2}$ Department of Chemical Engineering, Hanyang University, Seoul 133-791, Republic of Korea \\ ${ }^{3}$ Department of Polymer Science \& Technology, Pusan National University, Busan 609-735, Republic of Korea \\ ${ }^{4}$ Korea Research Institute of Chemical Technology, Daejeon 305-600, Republic of Korea \\ Correspondence should be addressed to Kwang-Sun Ryu, ryuks@ulsan.ac.kr
}

Received 17 February 2012; Revised 11 May 2012; Accepted 21 May 2012

Academic Editor: Luiz Antonio Ferreira Coelho

Copyright () 2012 Su-Ryeon Yun et al. This is an open access article distributed under the Creative Commons Attribution License, which permits unrestricted use, distribution, and reproduction in any medium, provided the original work is properly cited.

Polyaniline (Pani) and polypyrrole (Ppy) half hollow spheres with different shell thicknesses were successfully synthesized by three steps process using polystyrene (PS) as the core. The PS core was synthesized by emulsion polymerization. Aniline and pyrrole monomers were polymerized on the surface of the PS core. The shells of Pani and Ppy were fabricated by adding different amounts of aniline and pyrrole monomers. PS cores were dissolved and removed from the core shell structure by solvent extraction. The thicknesses of the Pani and Ppy half hollow spheres were observed by FE-SEM and FE-TEM. The chemical structures of the Pani and Ppy half hollow spheres were characterized by FT-IR spectroscopy and UV-Vis spectroscopy. The shell thicknesses of the Pani half hollow spheres were $30.2,38.0,42.2,48.2$, and $52.4 \mathrm{~nm}$, while the shell thicknesses of the Ppy half hollow spheres were 16.0 , 22.0, 27.0, and $34.0 \mathrm{~nm}$. The shell thicknesses of Pani and Ppy half hollow spheres linearly increased as the amount of the monomer increased. Therefore, the shell thickness of the Pani and Ppy half hollow spheres can be controlled in these ranges.

\section{Introduction}

Conducting polymers have recently received much attention due to their long conjugation lengths, metallic conductivity, and their promise for applications in molecular wires, nanoelectronics, optoelectronic devices, and biomedical devices [1-3]. Among the conjugated conducting polymers, Pani and Ppy have drawn the most attention due to their superior electroactivity, good electrical conductivity, and chemical stability. Owing to their physical, chemical, and electrochemical properties, Pani and Ppy have been applied in many different fields including sensors, electronics, semitransistors, flat-panel displays, cable shielding, ion-selective membranes, electrocatalysis, and as the cathode material in rechargeable batteries [4-6].

Pani and Ppy are known as an inherent electrically conductive polymer due to the conjugation of the single and double bonds alternating within the macromolecular architecture. The extra electrons of a double bond in a conjugated system are free to wander or move through the polymer chain, which induces electrical conductivity [710]. The conductivity of Pani and Ppy can be significantly improved by doping with oxidizing agents. During the oxidation process, an adequate amount of anions from the solutions with $\mathrm{HCl}$ and $\mathrm{FeCl}_{3} \cdot 6 \mathrm{H}_{2} \mathrm{O}$ is usually incorporated to compensate the electrogenerated positive charges (polaronic or bipolaronic species) in Scheme 1. The electrical conductivity of Pani and Ppy can be effectively increased to the level of a few tenths of S/cm through p-doping [11-13].

Recently, there has been immense interest in the fabrication of core-shell particles with unique and tailored properties for various applications in material sciences [14-16]. Among the core-shell particles, inorganic coated polymer (core-shell) capsules and hollow spheres have increasingly attracted interest because of their potential applications in catalysis, controlled delivery, artificial cells, light fillers, low dielectric constant materials, acoustic insulation, the paint industry, and photonic crystals. 
The latex sphere approach uses polystyrene spheres as a template, assisted by a template pretreatment process or layer-by-layer technique $[14,15]$. However, the hollow spheres that are obtained have smooth surfaces. Recently, Wan et al. developed an "emulsion template" approach to prepare hollow Pani spheres in an aqueous media using spherical micelles composed of a dopant and monomer as a template $[16,17]$. Otherwise, template spheres have been prepared by simple sulfonation of PS spheres or have been purchased for use. Pani and Ppy were polymerized by an electrochemical method. Moreover, the sizes of Pani and Ppy hollow spheres are generally widely dispersed, and the control of the morphology of Pani and Ppy is difficult. The PS cores are then removed by solvents, which are usually expensive and toxic organic solvents such as DMF, THF, chloroform, and toluene [15, 18-20].

In most of paper, hollow sphere structures were formatted by the layer-by-layer method. But in this paper, layerby-layer method was partly changed for surface modification of PS. This method was progressed with three steps. The PS templates were polymerized by micelle of anionic surfactant for using core. The surface of PS was directly modified by anion surfactant and formatted negative charge. The Pani and Ppy were evenly coated on surface-modified PS because doped Pani and Ppy have positive charge, respectively. And then, the PS cores were dissolved using organic solvent to produce Pani hollow sphere. This method is described in Scheme 1. We report an approach to make PS templates with the same size by polymerization using a chemical method. We prepared Pani and Ppy with a half hollow sphere structure and controlled the shell thickness. Since Pani and Ppy are well viscously aggregated, the thickness control of Pani and Ppy with a core-shell structure is not easy. Thus, we aimed to control the shell thickness in the core-shell or half hollow sphere structure. A specific amount of monomer was added to control the shell thickness of Pani and Ppy in the core-shell or half hollow sphere structure. We demonstrated the ability to obtain a desired shell thickness of Pani and Ppy half hollow spheres.

\section{Experimental}

Styrene (>99\%, Aldrich), aniline (>99.5\%, Aldrich), and pyrrole ( $98 \%$, Aldrich) monomers were purified by passing through aluminum oxide $(\sim 150$ mesh, $58 \AA)$ and distillation before being stored in a refrigerator. Potassium peroxodisulfate $\left(\mathrm{K}_{2} \mathrm{~S}_{2} \mathrm{O}_{8}\right.$, KPS, 99\%), ammonium persulfate $\left(\left(\mathrm{NH}_{4}\right)_{2} \mathrm{~S}_{2} \mathrm{O}_{8}\right.$, APS, 98\%) as initiator, dodecyl sulfate, sodium dodecyl sulfate $\left(\mathrm{C}_{12} \mathrm{H}_{25} \mathrm{O}_{4}\right.$ Sna, SDS, $\left.99 \%\right)$ as surfactant, divinylbenzene (DVB, 55\% mixture of isomers) as a cross-linking agent, hydrochloric acid $(\mathrm{HCl}, 37 \%)$, and iron(III) chloride hexahydrate $\left(\mathrm{FeCl}_{3} \cdot 6 \mathrm{H}_{2} \mathrm{O}, 97 \%\right)$ were used as received from Aldrich. Acetone was used as received from SK Chemical.

The PS was synthesized by emulsion polymerization. The styrene was refined by $\mathrm{Al}_{2} \mathrm{O}_{3}$ to remove the polymerization inhibitors. The $20 \mathrm{~g}(0.2 \mathrm{~mol})$ of styrene monomer and $0.04 \mathrm{~g}$ $\left(1.4 \times 10^{-4} \mathrm{~mol}\right)$ of SDS were dispersed in $180 \mathrm{~g}$ of deionized water, and, then, $0.2 \mathrm{~g}\left(7.3 \times 10^{-4} \mathrm{~mol}\right)$ of KPS was added under constant stirring and a $\mathrm{N}_{2}$ atmosphere at $80^{\circ} \mathrm{C}$ for $4 \mathrm{~h}$.

The $80 \mathrm{~mL}$ of the PS templates were diluted in $320 \mathrm{~mL}$ of deionized water to disperse the aniline monomer on the surface of the PS templates. Then, $1.2 \mathrm{~mL}\left(1.3 \times 10^{-2} \mathrm{~mol}\right)$ of aniline and $0.12 \mathrm{~mL}\left(4.6 \times 10^{-4} \mathrm{~mol}\right)$ of DVB were added into the PS emulsion and dispersed for $30 \mathrm{~min}$ in order to arrange the aniline monomer on the surfaces of the PS cores and to improve the stability of the resulting Pani shell. $1 \mathrm{M}$ $\mathrm{HCl}$ and APS were added to the solution mixture, and the polymerization of Pani proceeded at room temperature for $24 \mathrm{~h}$ (aniline: APS $=1: 1(\mathrm{~mol})$ ). The thickness of the Pani shell was adjusted by varying the amount of added aniline monomer among volumes of $1.2,2.4,3.6,4.8$, and $6.0 \mathrm{~mL}$, while $0.12,0.24,0.36,0.48$, and $0.60 \mathrm{~mL}$ of DVB was added, respectively. Therefore, the aniline monomer was added at ratios of $1,2,3,4$, and 5 times the base volume of $1.2 \mathrm{~mL}$ in the different experiments. Green PS/Pani composite spheres with a core-shell structure were produced and purified by centrifugation and dried in a vacuum oven at $60^{\circ} \mathrm{C}$.

PS/Ppy with a core-shell structure was prepared similarly to the PS/Pani with a core-shell structure. $0.6 \mathrm{~mL}(8.47 \times$ $\left.10^{-3} \mathrm{~mol}\right)$ of pyrrole and $0.06 \mathrm{~mL}\left(2.3 \times 10^{-4} \mathrm{~mol}\right)$ of DVB were added into the PS emulsion and dispersed for $30 \mathrm{~min}$. Then, $4.5 \mathrm{~g}\left(1.6 \times 10^{-2} \mathrm{~mol}\right)$ of $\mathrm{FeCl}_{3} \cdot 6 \mathrm{H}_{2} \mathrm{O}$ was dispersed in $25 \mathrm{~mL}$ of deionized water. The $\mathrm{FeCl}_{3} \cdot 6 \mathrm{H}_{2} \mathrm{O}$ solution and $1.97 \mathrm{~g}\left(8.5 \times 10^{-3} \mathrm{~mol}\right)$ of APS were added to the solution mixture, and the polymerization of Ppy proceeded at room temperature for $24 \mathrm{~h}$ (pyrrole: APS $=1: 1(\mathrm{~mol})$ ). The thickness of the Ppy shell was varied by adjusting the amount of added pyrrole monomer among volumes of $0.6,1.2,1.8$, and $2.4 \mathrm{~mL}$, while $0.06,0.12,0.18$, and $0.24 \mathrm{~mL}$ of DVB was added, respectively. Therefore, the pyrrole monomer was added at ratios of $1,2,3$, and 4 times the base volume of $0.6 \mathrm{~mL}$. Black PS/Ppy composite spheres were produced and purified by centrifugation and dried in a vacuum oven at $60^{\circ} \mathrm{C}$.

To prepare the Pani and Ppy half hollow spheres, excess acetone was added to the dried PS/Pani and PS/Ppy core shells with different thicknesses at room temperature. The PS cores were dissolved in acetone to produce Pani and Ppy half hollow spheres over $48 \mathrm{~h}$. The Pani and Ppy half hollow spheres were washed several times with deionized water to remove the initiator, unreacted monomer, and oligomer. Finally, Pani and Ppy half hollow spheres with different thicknesses were dried in a vacuum oven at $60^{\circ} \mathrm{C}$. The full preparation processes of the Pani and Ppy half hollow spheres are schematically illustrated in Scheme 1.

The Pani and Ppy core shell and half hollow spheres with different shell thicknesses were analyzed by Fourier transform infrared spectroscopy (FT-IR) recorded on Varian 2000 in the range from 400 to $3,250 \mathrm{~cm}^{-1}$. We used the pellet type using $\mathrm{KBr}$ powder for measuring. And the optical properties of prepared samples characterized through ultraviolet visible spectroscopy (UV-Vis, Varian Karry) in the range of $250-900 \mathrm{~nm}$. The NMP ( $\mathrm{N}$-methyl pyrrolidinone) was used as solvent for Pani. The surface morphologies and shell thicknesses were observed by field emission scanning electron microscopy (FE-SEM, Zeiss Supra-40) with an 


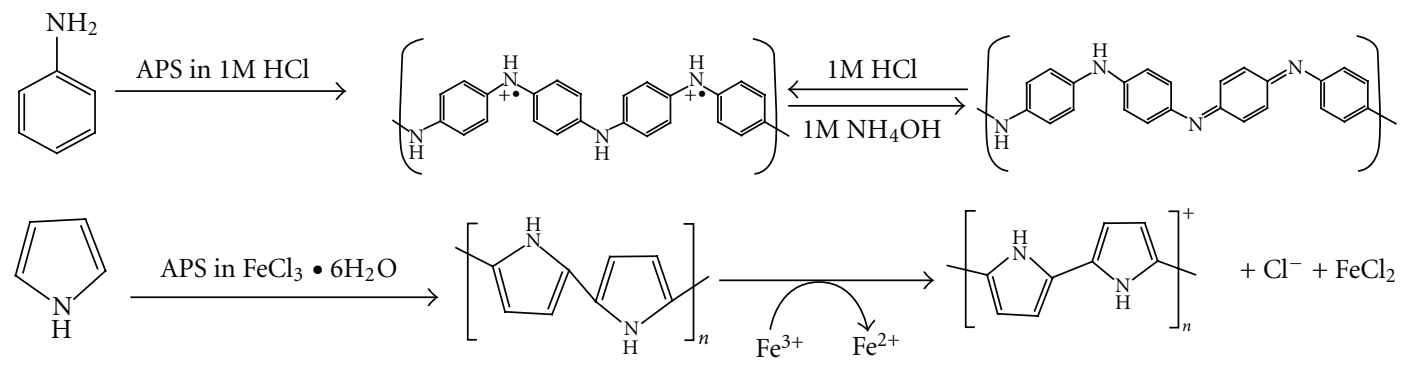

Scheme 1: The oxidation of aniline and pyrrole with ammonium persulfate in dopant solution for doping and dedoping.

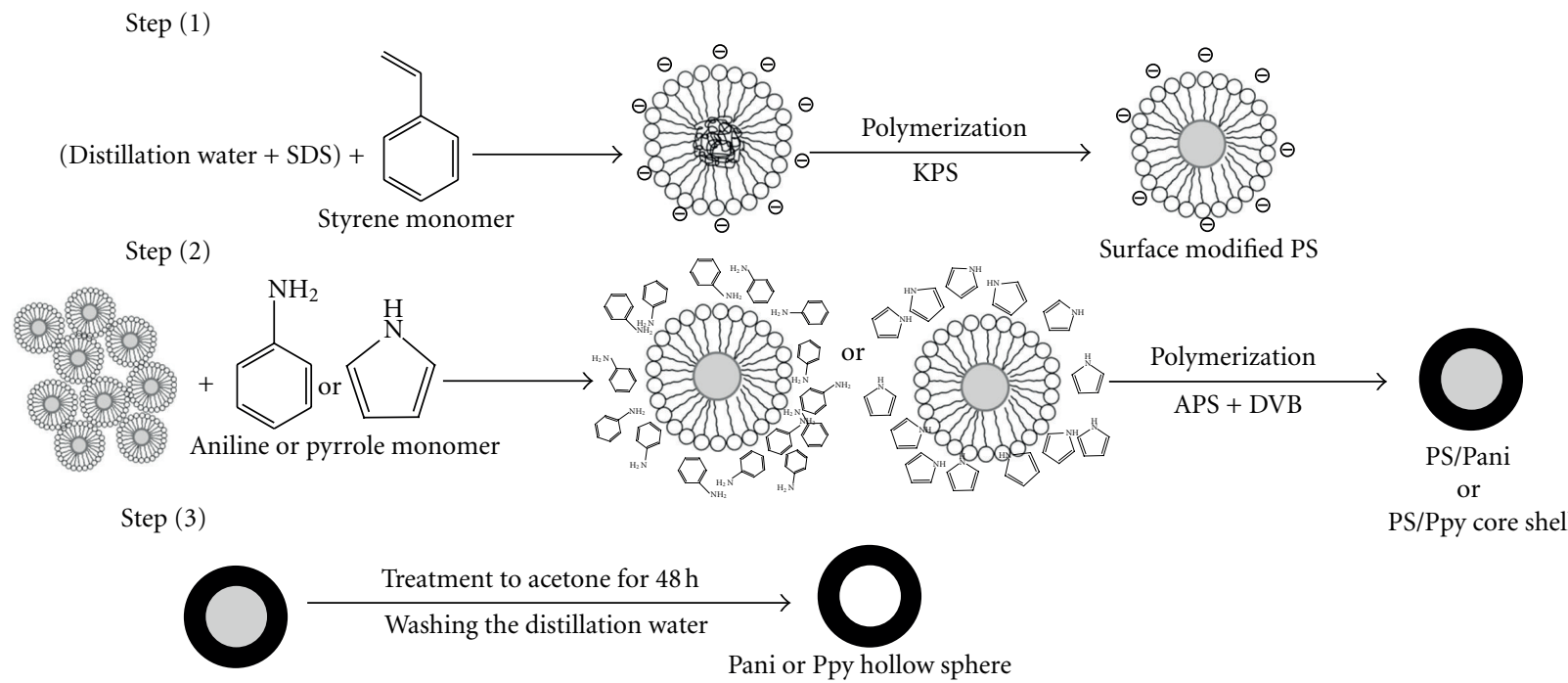

Scheme 2: Schematic illustration of the three-steps procedure used to produce Pani or Ppy half hollow spheres with different shell thicknesses.

accelerating voltage of $15 \mathrm{kV}$ and field emission transmission electron microscopy (FE-TEM, JEM 2100F, JEOL operated at $200 \mathrm{kV})$.

\section{Results and Discussion}

The FT-IR spectra of the overall process materials are shown in Figure 1. Figure 1(a) shows the FT-IR spectra of PS, bulk Pani, and Pani half hollow spheres. The characteristic absorption bands due to the PS component are at around 1496, 1449, 754, and $696 \mathrm{~cm}^{-1}$. The bands at 1,496 and $1,449 \mathrm{~cm}^{-1}$ are indicative of the $\mathrm{C}=\mathrm{C}$ ring stretching and $\mathrm{C}-\mathrm{H}$ stretching of $\mathrm{PS}$, respectively [21]. The peaks of $\mathrm{C}=\mathrm{N}$ and $\mathrm{C}=\mathrm{C}$ stretching of quinoid and benzenoid rings are specific peaks of the bulk Pani. Similar FT-IR spectra to those shown in Figure 1(a) were observed for the bulk Pani and Pani half hollow spheres. The Pani half hollow spheres exhibited peaks at $1,580,1,494,1,302,1,142$, and $692 \mathrm{~cm}^{-1}$. The peaks at 1,580 and $1,494 \mathrm{~cm}^{-1}$ indicated the $\mathrm{C}=\mathrm{N}$ and $\mathrm{C}=\mathrm{C}$ stretching mode of the quinoid and benzenoid rings of the Pani half hollow spheres, respectively. The peaks of at 1,302 and $1,142 \mathrm{~cm}^{-1}$ corresponded to $\mathrm{C}-\mathrm{N}$ stretching
( $-\mathrm{N}$-benzenoid-N-) and $\mathrm{C}=\mathrm{N}$ stretching $(-\mathrm{N}=$ quinoid $=\mathrm{N}-$ ), respectively [22].

Figure 1(b) shows the FT-IR spectra of PS, bulk Ppy, and Ppy half hollow spheres with different thicknesses. Similar FT-IR spectra were observed for the bulk Ppy and Ppy half hollow spheres. The Ppy half hollow spheres shown in Figure 1(b) demonstrated peaks at 1,710, 1,157, 1,449, $1,371,1,192,1,046,978,753$, and $698 \mathrm{~cm}^{-1}$. The peak corresponding to oxygen dopant $\left(\mathrm{FeCl}_{3} \cdot 6 \mathrm{H}_{2} \mathrm{O}\right)$ occurred at $1,710 \mathrm{~cm}^{-1}$, and the two peaks of 1,449 and $1,192 \mathrm{~cm}^{-1}$ corresponded to the $\mathrm{C}-\mathrm{N}$ stretching. The peak observed at $1,557 \mathrm{~cm}^{-1}$ corresponded to the $\mathrm{C}-\mathrm{C}$ stretching peak observed from the pyrrole ring. The peaks at 1,371 and $1,046 \mathrm{~cm}^{-1}$ correspond to the $=\mathrm{C}-\mathrm{H}$ bond. The $918 \mathrm{~cm}^{-1}$ peak indicated the $\mathrm{C}-\mathrm{H}$ bending. The regions of the $\mathrm{C}-\mathrm{H}$ out-of-plane deformation vibration of the ring and the C-C out-of-plane ring deformation are indicated at 753 and $698 \mathrm{~cm}^{-1}$, respectively [23]. However, the characteristic absorption peaks of PS still existed in Pani and Ppy half hollow spheres at $760-700 \mathrm{~cm}^{-1}$. Therefore, we could guess that PS still remained in Pani and Ppy half hollow spheres.

Figures 2(a) and 2(b) show the UV-Vis spectra of the Pani half hollow spheres and Ppy half hollow spheres, respectively. The Pani half hollow spheres in Figure 2(a) show 


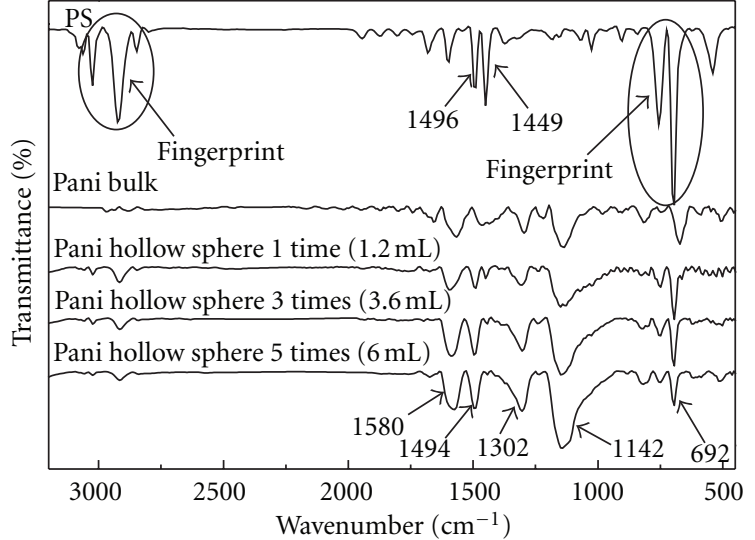

(a)

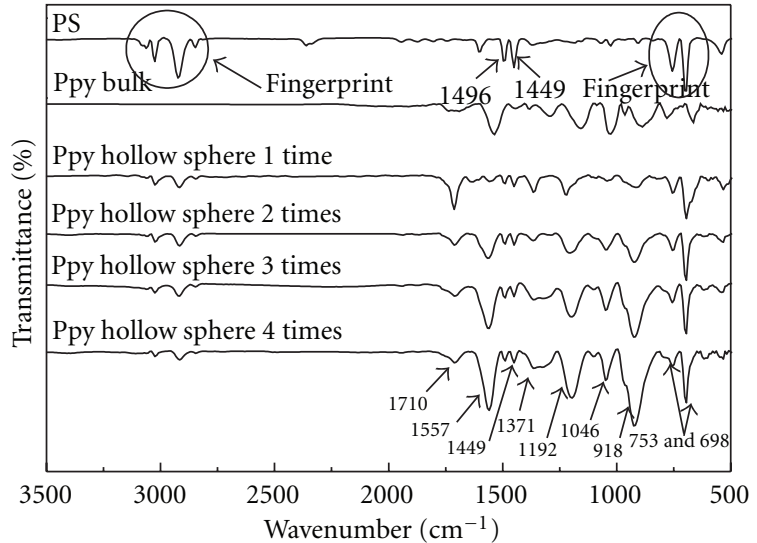

(b)

Figure 1: FT-IR spectra of (a) Pani half hollow spheres and (b) Ppy half hollow spheres with different thicknesses.

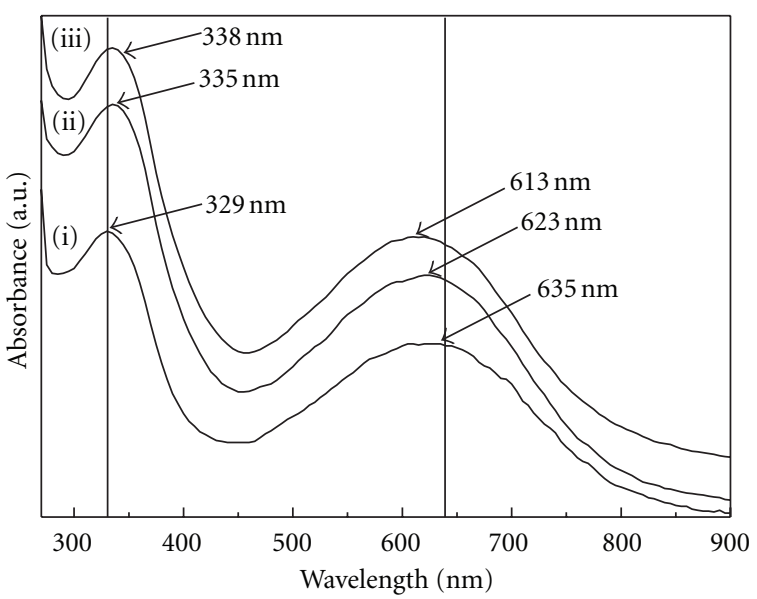

(a)

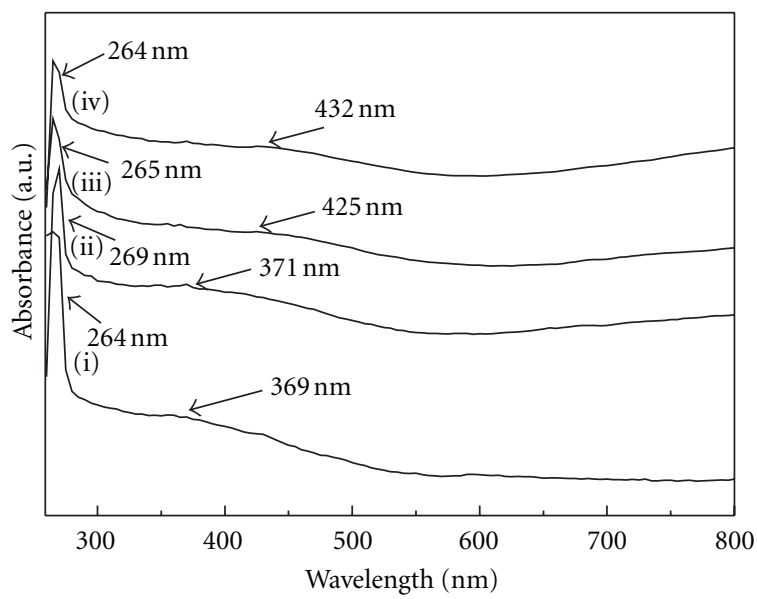

(b)

Figure 2: UV-Vis spectra of (a) Pani half hollow spheres produced with (i) 1.2, (ii) 3.6, and (iii) $6.0 \mathrm{~mL}$ of aniline and (b) Ppy half hollow spheres made with (i) 0.6 , (ii) 1.2 , (iii) 1.8 , and (iv) $2.4 \mathrm{~mL}$ of pyrrole.

two characteristic absorption broad band peaks at around 330 and $620 \mathrm{~nm}$. The peaks at 329, 335, and $338 \mathrm{~nm}$ can be ascribed to the $\pi-\pi^{*}$ transition in the benzenoid rings, and the peaks at 635,623 , and $613 \mathrm{~nm}$ can be attributed to the polaron-polaron transition. As the amount of the aniline monomer was increased, the $\pi-\pi^{*}$ transition red shifted and the polaron-polaron transition blue shifted.

The $\pi-\pi^{*}$ transitions shown in Figure 2(a) were red shifted to longer wavelengths. Thus, the thicknesses of the Pani half hollow spheres were larger, and the $\pi-\pi^{*}$ transition peaks appeared at 329,335 , and $338 \mathrm{~nm}$. The red-shift is indicative of small changes of the chain geometry interring the torsion angle along the Pani backbone leading to mixing of the $\pi$ and $n$ orbital of the Pani chain and dopant. The red shifted transition of the $\pi-\pi^{*}$ band gap indicates a reduction of the band gap, allowing the phenylene rings to adopt a more twisted geometry. As the thickness of the Pani hollow spheres increased, the polaron-polaron peaks were shifted to shorter wavelengths. The position of the shifted polaron-polaron peaks varied depending on the experimental conditions employed. The blue shifting was normally accompanied by the appearance and growth of a shoulder on the short wavelength side of the absorption band in the UV-Vis spectra (Figure 2(a)). The absorption of the polaron-polaron transition was observed at 635,623 , and $613 \mathrm{~nm}$. This blue shift was dependent on the thickness. It has been proposed that direct interaction of planar benzenoid and quinoid chromophore groups rather than oxidation of the polymer chains is responsible for the blue-shifted exciton transition in Pani half hollow spheres with different thicknesses.

The Ppy half hollow spheres in Figure 2(b) show absorption peaks at 264,269,265, and $264 \mathrm{~nm}$ can be ascribed to the $\pi-\pi^{*}$ transition in the aromatic ring, and the peaks at $369,371,425$, and $432 \mathrm{~nm}$ can be attributed to the $\pi-\pi^{*}$ transition.

The structures, morphologies, and diameter of particles were investigated by FE-SEM and FE-TEM. The FE-SEM images in Figure 3 show the successful formation of uniform 

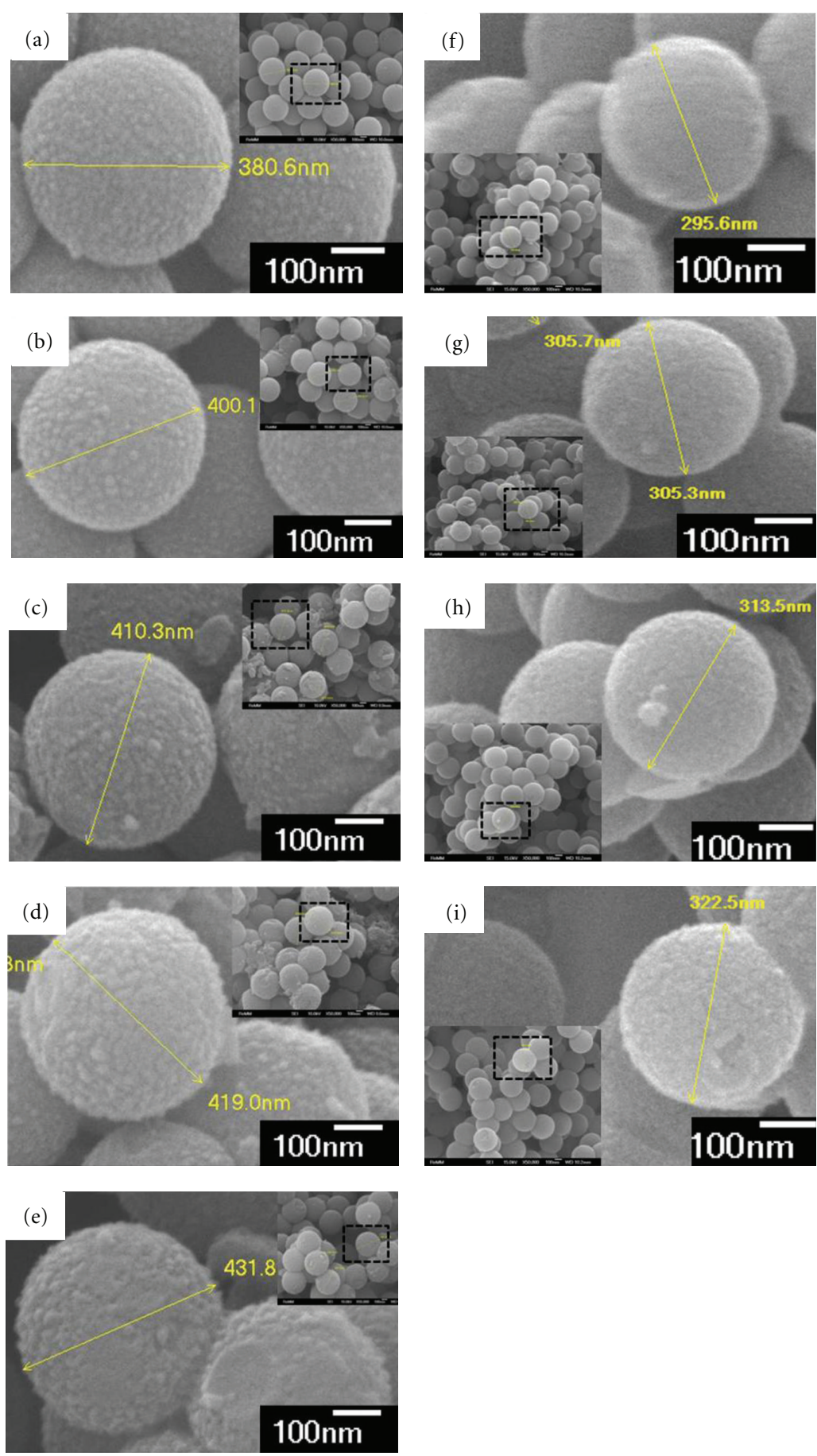

FIGURE 3: FE-SEM images of the PS/Pani core shell produced with (a) 1.2, (b) 2.4, (c) 3.6, (d) 4.8, and (e) $6.0 \mathrm{~mL}$ of aniline and the PS/Ppy core shell made with (f) 0.6, (g) 1.2 , (h) 1.8 , and (i) $2.4 \mathrm{~mL}$ of pyrrole.

Pani shells on PS cores (Figures 3(a), 3(b), 3(c), 3(d), and 3(e)) and Ppy shells on PS cores (Figures 3(f), 3(g), 3(h), and 3(i)). In the experiments, we used PS cores with the same diameter. These images reveal that the PS/Pani and PS/Ppy core shells have a rough surface. The diameters of the PS/Pani core shell in Figures 3(a), 3(b), 3(c), 3(d), and 3 (e) were measured to be 380.6, 400.1, 410.3, 419.0, and $431.8 \mathrm{~nm}$, respectively. The diameters of the PS/Ppy core shell in Figures 3(f), 3(g), 3(h), and 3(i) were 295.6, 305.3, 313.5, and $322.5 \mathrm{~nm}$, respectively. As the amount of the monomer increased, the shell diameters of PS/Pani and PS/Ppy also increased.

The structure and thickness of the Pani half hollow spheres were investigated by FE-SEM and FE-TEM. FE-SEM and FE-TEM images of the Pani half hollow spheres and Ppy half hollow spheres after removal of the PS core are 

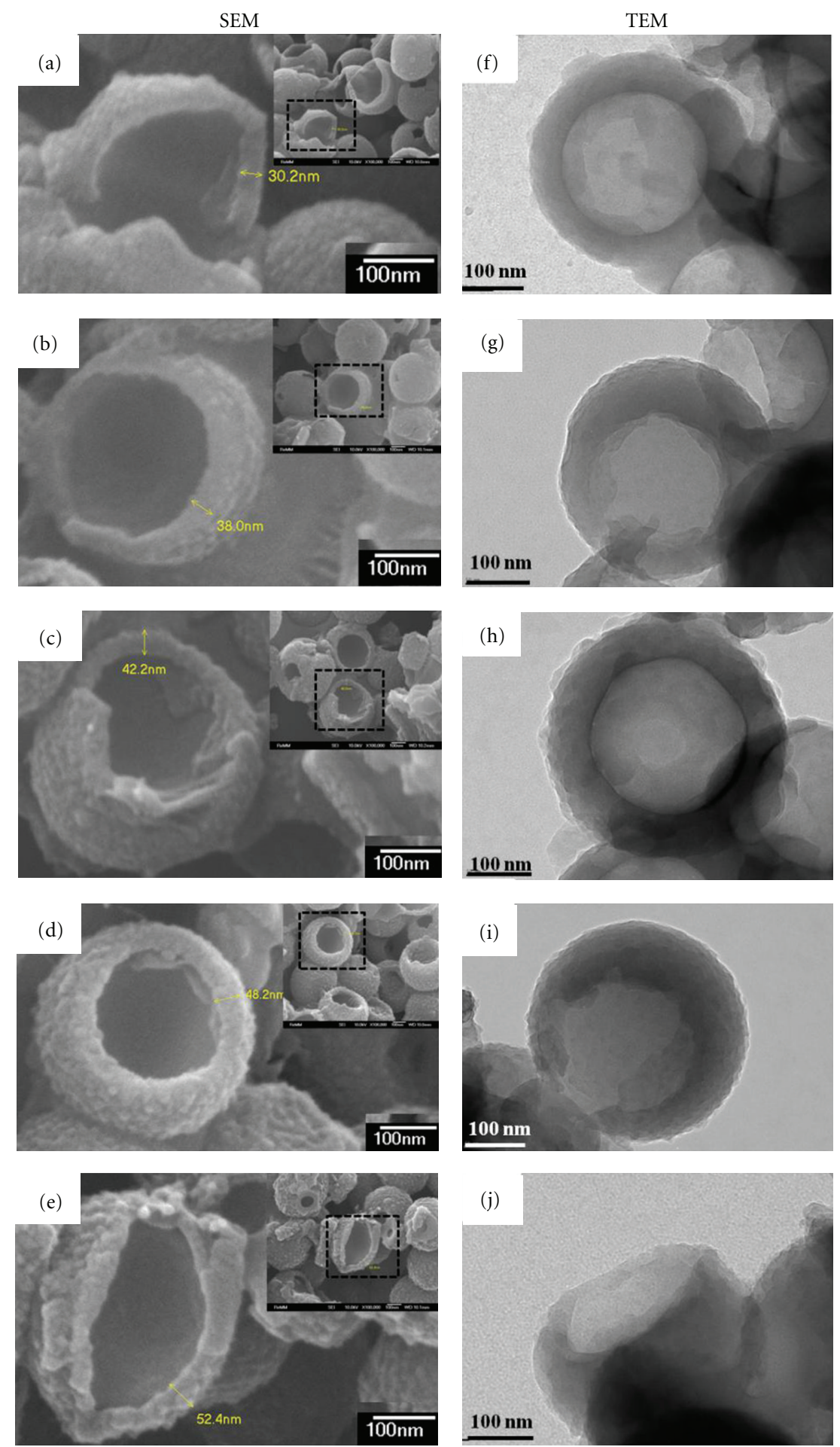

Figure 4: FE-SEM and FE-TEM images of Pani half hollow spheres made with (a, f) 1.2, (b, g) 2.4, (c, h) 3.6, (d, i) 4.8, and (e, j) 6.0 mL of aniline.

shown in Figures 4 and 5, respectively. Therefore, the half hollow spheres had vacancies, as observed in the FE-TEM image. When the PS was treated with acetone, the acetone permeated the shell (Pani or Ppy) and melted the PS. Next, the melted PS got out of the shell. At this time, the melted
PS got out from the part of weak or thin shell. After enough time had passed, the entrance was enlarged.

The thickness of the half hollow spheres was investigated by FE-SEM. The FE-SEM images of the Pani half hollow spheres (Figures 4(a), 4(b), 4(c), 4(d), and 4(e)) and Ppy 

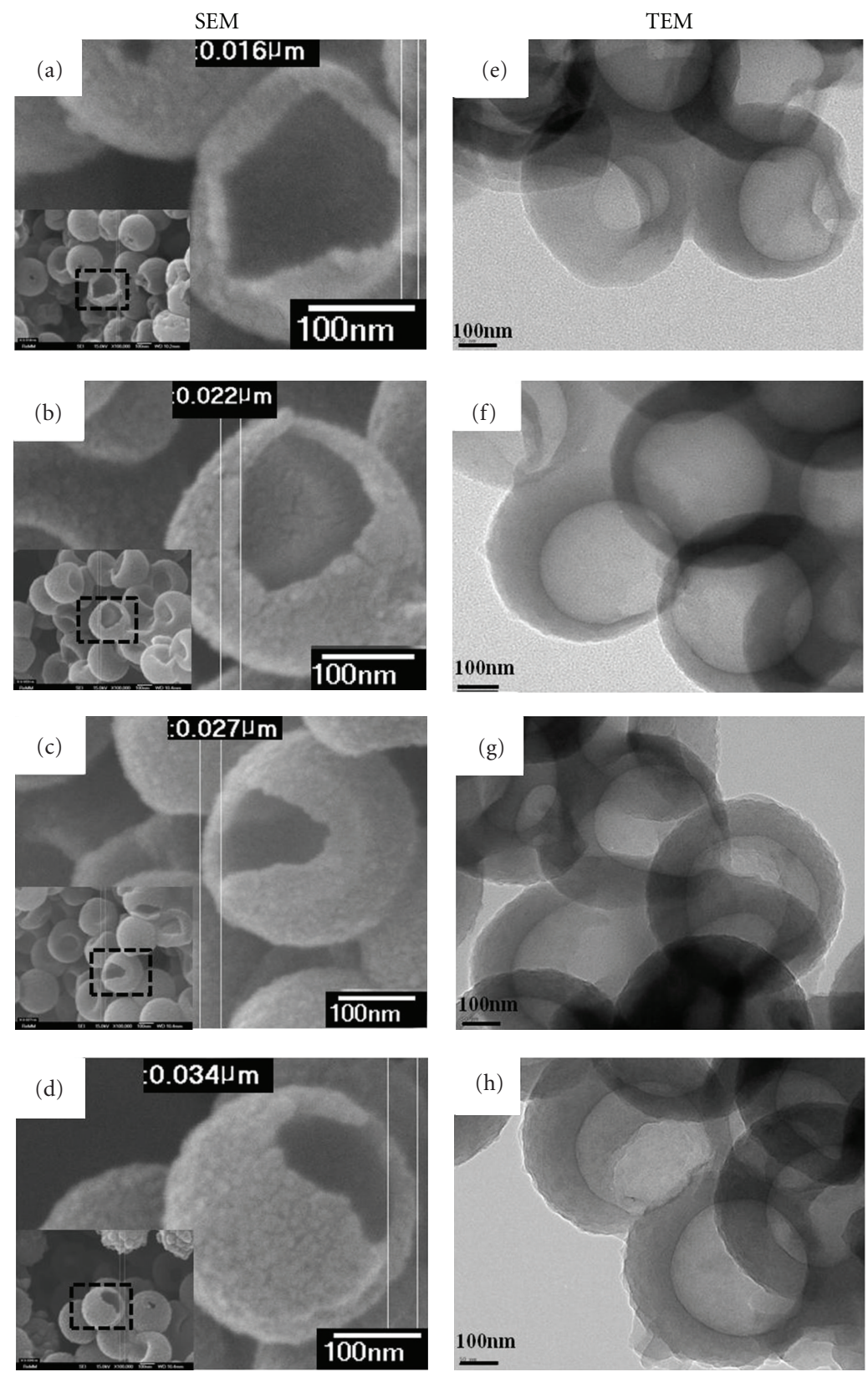

Figure 5: FE-SEM and FE-TEM images of Ppy half hollow spheres produced with (a, e) 0.6, (b, f) 1.2, (c, g) 1.8, and (d, h) $2.4 \mathrm{~mL}$ of pyrrole.

half hollow spheres (Figures 5(a), 5(b), 5(c), and 5(d)) demonstrated different thicknesses. The thicknesses of the Pani half hollow spheres (Figures 4(a), 4(b), 4(c), 4(d), and $4(\mathrm{e})$ ) were $30.2,38.0,42.2,48.2$, and $52.4 \mathrm{~nm}$, respectively. The thicknesses of the Ppy half hollow spheres (Figures 5(a), $5(\mathrm{~b}), 5(\mathrm{c})$, and $5(\mathrm{~d}))$ were $16.0,22.0,27.0$, and $34.0 \mathrm{~nm}$, respectively.

Figures 6(a) and 6(b) show the shell thicknesses of the Pani half hollow spheres and Ppy half hollow spheres as a function of the amount of monomer. The shell thickness linearly increased with increasing amount of monomer. From these results, it is possible to control the shell thickness of the Pani and Ppy half hollow spheres by adjusting the amount of aniline or pyrrole monomer. Therefore, it is able to control the desired shell thickness of the Pani and Ppy half hollow sphere in these ranges. As demonstrated in the results shown in Figures 6(a) and 6(b), it is simple to obtain Pani and Ppy half hollow spheres with specific shell thicknesses.

\section{Conclusions}

Pani and Ppy half hollow spheres with a controlled thickness were successfully synthesized by three steps using the PS core with micelle assistance. The PS was polymerized by emulsion polymerization using an anionic surfactant with 


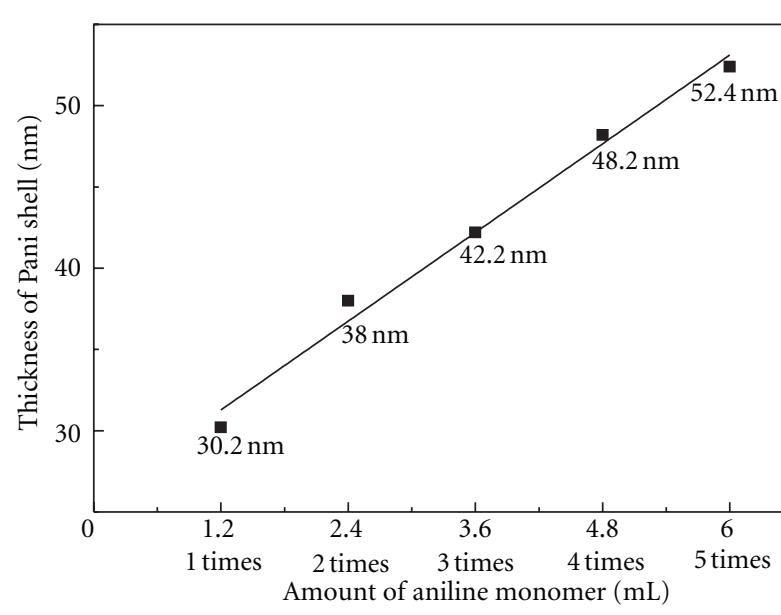

(a)

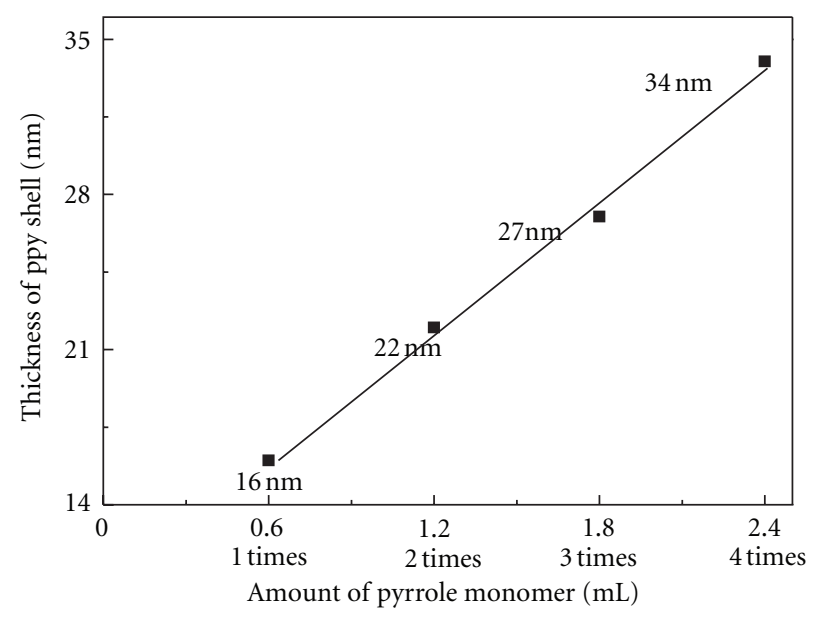

(b)

Figure 6: The shell thicknesses of the (a) Pani half hollow spheres and (b) Ppy half hollow spheres as a function of the amount of added monomer.

SDS for surface modification. Aniline and pyrrole were polymerized by an oxidizing agent on the PS core surfaces. The shell thickness of the Pani and Ppy was controlled by adjusting the amount of aniline and pyrrole monomer, respectively. In separate experiments, aniline monomer was added at volumes of 1.2, 2.4, 3.6, 4.8, and $6.0 \mathrm{~mL}$, and pyrrole monomer was added at volumes of $0.6,1.2,1.8$, and $2.4 \mathrm{~mL}$. Finally, the PS was dissolved in acetone to create Pani and Ppy half hollow spheres. The shell thicknesses of the Pani and Ppy in the half hollow sphere structure were 30.2, 38.0, 42.2, 48.2, and $52.4 \mathrm{~nm}$ and 16.0, 22.0, 27.0, and $34.0 \mathrm{~nm}$, respectively. The shell thickness of the Pani and Ppy was proportional to the amount of aniline and pyrrole monomer. Therefore, it is possible to control the desired shell thickness of the Pani and Ppy half hollow spheres in these ranges.

\section{Acknowledgments}

This research was supported by a grant from the Fundamental R\&D Program for Core Technology of Materials funded by the Ministry of Knowledge Economy, Republic of Korea.

\section{References}

[1] J. Lei, V. P. Menon, and C. R. Martin, "Chemical preparation of conductive polypyrrole-polytetrafluoroethene composites," Polymers for Advanced Technologies, vol. 4, no. 2-3, pp. 124$132,1994$.

[2] Y. Yang and M. Wan, "Chiral nanotubes of polyaniline synthesized by a template-free method," Journal of Materials Chemistry, vol. 12, no. 4, pp. 897-901, 2002.

[3] Z. Chen, Y. Takei, B. A. Deore, and T. Nagaoka, "Enantioselective uptake of amino acid with overoxidized polypyrrole colloid templated with L-lactate," Analyst, vol. 125, no. 12, pp. 2249-2254, 2000.

[4] J. Chen, A. K. Burrell, G. E. Collis et al., "Preparation, characterisation and biosensor application of conducting polymers based on ferrocene substituted thiophene and terthiophene," Electrochimica Acta, vol. 47, no. 17, pp. 2715-2724, 2002.
[5] J. Chen, C. O. Too, G. G. Wallace, G. F. Swiegers, B. W. Skelton, and A. H. White, "Redox-active conducting polymers incorporating ferrocenes. Preparation, characterization and bio-sensing properties of ferrocenylpropyl and butyl polypyrroles," Electrochimica Acta, vol. 47, no. 26, pp. 42274238, 2002.

[6] J. Chen, J. Huang, G. F. Swiegers, C. O. Too, and G. G. Wallace, "A readily-prepared electrocatalytic coating that is more active than platinum for hydrogen generation in $1 \mathrm{M}$ strong acid," Chemical Communications, vol. 10, no. 3, pp. 308-309, 2004.

[7] O. D. Velev, T. A. Jede, R. F. Lobo, and A. M. Lenhoff, "Porous silica via colloidal crystallization,” Nature, vol. 389, no. 6650, pp. 447-448, 1997.

[8] B. T. Holland, C. F. Blanford, and A. Stein, "Synthesis of macroporous minerals with highly ordered three-dimensional arrays of spheroidal voids," Science, vol. 281, no. 5376, pp. 538$540,1998$.

[9] F. Caruso, R. A. Caruso, and H. Möhwald, "Nanoengineering of inorganic and hybrid hollow spheres by colloidal templating," Science, vol. 282, no. 5391, pp. 1111-1114, 1998.

[10] W. Schärtl, "Crosslinked spherical nanoparticles with coreshell topology," Advanced Materials, vol. 12, no. 24, pp. 18991908, 2000.

[11] F. Caruso, "Nanoengineering of particle surfaces," Advanced Materials, vol. 13, no. 1, pp. 11-22, 2001.

[12] Y. Zhang, Z. Huang, F. Tang, and J. Ren, "Ferrite hollow spheres with tunable magnetic properties," Thin Solid Films, vol. 515, no. 4, pp. 2555-2561, 2006.

[13] W. Zhao, H. Chen, Y. Li, A. Li, M. Lang, and J. Shi, "Uniform rattle-type hollow magnetic mesoporous spheres as drug delivery carriers and their sustained-release property," Advanced Functional Materials, vol. 18, no. 18, pp. 2780-2788, 2008.

[14] M. K. Park, K. Onishi, J. Locklin, F. Caruso, and R. C. Advincula, "Self-assembly and characterization of polyaniline and sulfonated polystyrene multilayer-coated colloidal particles and hollow shells," Langmuir, vol. 19, no. 20, pp. 8550-8554, 2003.

[15] Z. Niu, Z. Yang, Z. Hu, Y. Lu, and C. C. Han, "Polyaniline-silica composite conductive capsules and hollow spheres," Advanced Functional Materials, vol. 13, no. 12, pp. 949-954, 2003. 
[16] Z. Wei and M. Wan, "Hollow microspheres of polyaniline synthesized with an aniline emulsion template," Advanced Materials, vol. 14, no. 18, pp. 1314-1317, 2002.

[17] L. Zhang and M. Wan, "Self-assembly of polyaniline from nanotubes to hollow microspheres," Advanced Functional Materials, vol. 13, no. 10, pp. 815-820, 2003.

[18] K. Huang, X. H. Meng, and M. Wan, "Polyaniline hollow microspheres constructed with their own self-assembled nanofibers," Journal of Applied Polymer Science, vol. 100, no. 4, pp. 3050-3054, 2006.

[19] Z. Yang, Z. Niu, Y. Lu, Z. Hu, and C. C. Han, "Templated synthesis of inorganic hollow spheres with a tunable cavity size onto core-shell gel particles," Angewandte Chemie, vol. 42, no. 17, pp. 1943-1945, 2003.

[20] X. Wang, J. Liu, X. Feng, M. Guo, and D. Sun, "Fabrication of hollow $\mathrm{Fe}_{3} \mathrm{O}_{4}$-polyaniline spheres with sulfonated polystyrene templates," Materials Chemistry and Physics, vol. 112, no. 2, pp. 319-321, 2008.

[21] X. Zhu, M. Elomaa, F. Sundholm, and C. H. Lochmüller, "Infrared and thermogravimetric studies of thermal degradation of polystyrene in the presence of ammonium sulfate," Polymer Degradation and Stability, vol. 62, no. 3, pp. 487-494, 1998.

[22] N. S. Sariciftci, H. Kuzmany, H. Neugebauer, and A. Neckel, "Structural and electronic transitions in polyaniline: a Fourier transform infrared spectroscopic study," The Journal of Chemical Physics, vol. 92, no. 7, pp. 4530-4539, 1990.

[23] R. G. Davidson and T. G. Turner, "An IR spectroscopic study of the electrochemical reduction of polypyrrole doped with dodecylsulfate anion," Synthetic Metals, vol. 72, no. 2, pp. 121$128,1995$. 

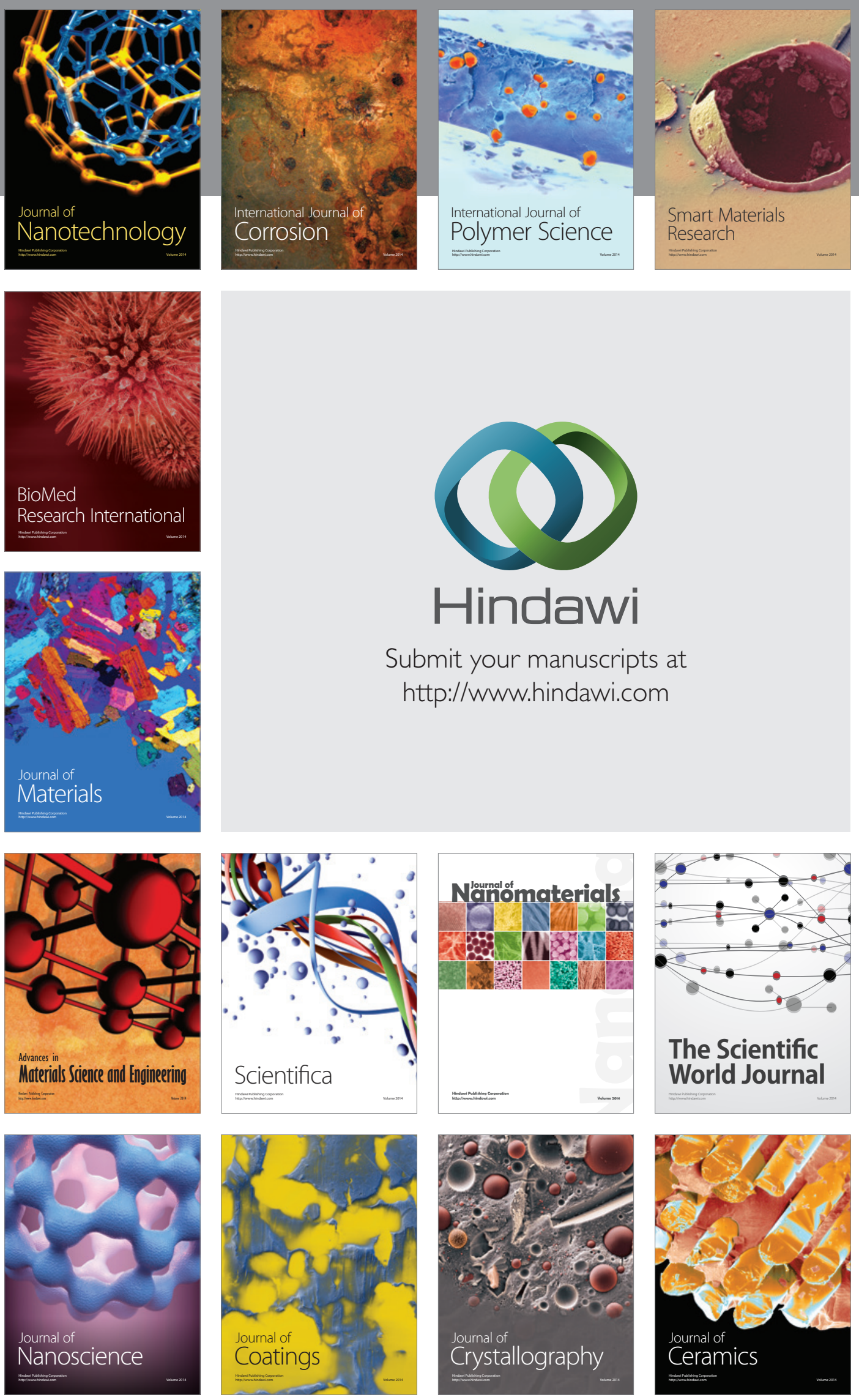

The Scientific World Journal

Submit your manuscripts at

http://www.hindawi.com

\section{World Journal}

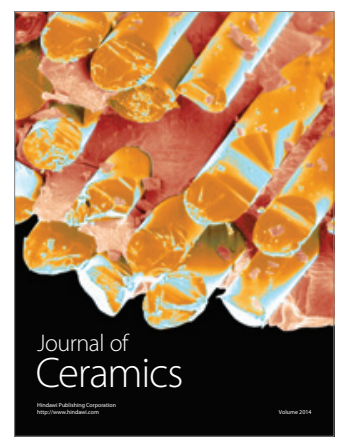

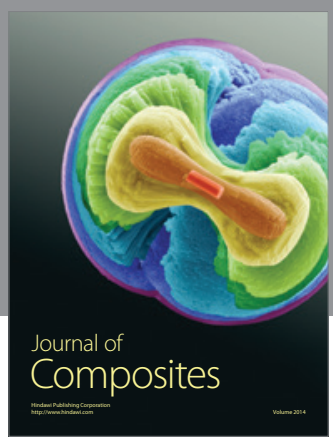
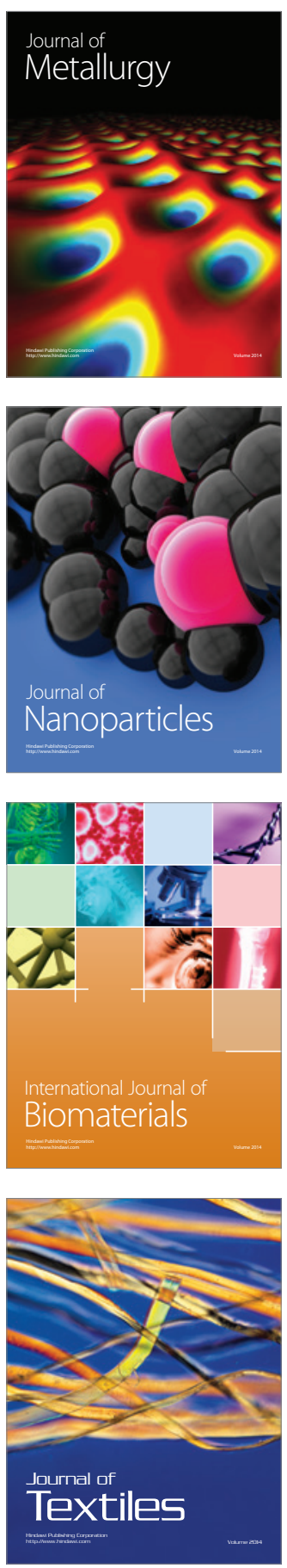